\title{
Prof. Dr. rer. nat. habil. Günter Hoppe zum 85. Geburtstag
}

\section{Hans-Joachim Bautsch ${ }^{1}$ \& Hans-Peter Schultze ${ }^{2}$}

Die ersten Kontakte mit dem Museum für Naturkunde hatte Günter Hoppe bereits vor über fünfundfünfzig Jahren. Er besuchte Paul Ramdohr, der damals noch, bis zu seiner Berufung im April 1950 nach Heidelberg, Direktor des Mineralogisch-Petrographischen Institutes und Museums war. Zu dieser Zeit war Günter Hoppe Student an der Universität Halle und mit seiner Abschlussarbeit beschäftigt. Sein Lehrer in Halle war Ferdinand von Wolff, noch im hohen Alter Direktor des Mineralogisch-Petrographischen Institutes. Begonnen hatte Günter Hoppe 1938 ein Studium der Chemie in Halle, das durch die Einberufung zum Militärdienst unterbrochen werden musste. Nach Rückkehr aus dem Krieg nahm er mit Beginn des Studienbetriebes an der Universität Halle 1946 sein Studium wieder auf, jetzt aber in der Mineralogie, wohl angeregt durch eine Praktikumstätigkeit in einem Bergbaubetrieb.

\section{Der Wissenschaftler}

Mit der Dissertation „Die akzessorischen Schwermineralien in Eruptivgesteinen am Beispiel des Ramberggranites und anderer Harzer Gesteine" (1950) begründete Günter Hoppe ein über fast zwei Jahrzehnte erfolgreich bearbeitetes Forschungsthema. Die dabei entwickelten präparativen und mikroskopischen Untersuchungsmethoden einer sehr detaillierten Erfassung und Charakterisierung der morphologischen Erscheinungen, insbesondere der akzessorischen Zirkone, führten zu wesentlichen gesteinsgenetischen Aussagen. Die zusammenfassenden und grundlegenden Darstellungen zur Methodik und zu den erzielbaren wissenschaftlichen Ergebnissen in der Habilitationsschrift „Die Verwendbarkeit morphologischer Erscheinungen an akzessorischen Zirkonen für petrogenetische Auswertungen“"
(Halle 1960) fanden, angewandt sowohl an magmatischen als auch an sedimentären und metamorphen Gesteinen, in zahlreichen weiteren wissenschaftlichen Arbeiten und auch anderweitig ihre Bestätigung und Anerkennung.

Im Jahre 1961 wurde Günter Hoppe zum Professor mit vollem Lehrauftrag für Mineralogie und Petrographie an die Universität Greifswald berufen. Ein Jahr später wurde er dort Direktor des Mineralogisch-Petrographischen Institutes. Hier in Greifswald ergab sich ein weiterer Schwerpunkt der wissenschaftlichen Forschungstätigkeit von Günter Hoppe, die Tonmineralogie. Die wissenschaftlichen Ergebnisse auf diesem Gebiet angewandter und genetischer Tonmineralogie sind umfangreich in den Arbeiten seiner Schüler und Mitarbeiter an der Greifswalder Universität dokumentiert. Kollegiale Anerkennung erhielt Günter Hoppe mit der Herausgabe und der Übernahme der Redaktion einer mineralogischen Zeitschrift. Die „Berichte der Deutschen Gesellschaft für Geologische Wissenschaften, Reihe B - Mineralogie und Lagerstättenforschung" erschienen im Akademie-Verlag ab 1965 bis zur Einstellung 1972.

\section{Der Wissenschaftshistoriker}

Im Zuge der Hochschulreform, die zu einschneidenden Veränderungen und negativen Auswirkungen insbesondere bei den mineralogischen Instituten der DDR führte, wurde Günter Hoppe im Herbst 1968 auf eine Professur für Mineralogie und Petrographie an der Humboldt-Universität und im folgenden Jahr als Direktor des Bereiches Mineralogie des Museums für Naturkunde berufen. Das Institut für Mineralogie (Kristallographie, Petrographie, Lagerstättenkunde) und Museum unter Will Kleber existierte nicht mehr. Es war in den Bereich Mineralogie

\footnotetext{
${ }^{1}$ Institut für Mineralogie, Museum für Naturkunde, Humboldt Universität zu Berlin, Invalidenstr. 43, D-10115 Berlin, Germany.

${ }_{2}^{2}$ Institut für Paläontologie, Museum für Naturkunde, Humboldt Universität zu Berlin, Invalidenstr. 43, D-10115 Berlin, Germany.

Erhalten Mai 2004, angenommen Juni 2004
} 
am Museum für Naturkunde und den Bereich Kristallographie der Sektion Physik geteilt worden. Zum ersten gehörten die Sammlungen, zum zweiten die Studenten, die Mitarbeiter und die Geräte. So fand Günter Hoppe bei seiner Berufung eine Situation vor, bei der nur die Zuwendung zur musealen Aufgabe übrig blieb, was im übrigen auch gefordert wurde. Und es ist außerordentlich, was seitdem von ihm in dieser Hinsicht in den Sammlungen, bei den Ausstellungen und auf dem Gebiet der Wissenschaftsgeschichte geleistet wurde.

Am Beginn stand der Aufbau einer stark historisch orientierten Meteoritenausstellung, wie sie in den wesentlichen Zügen noch heute im Ausstellungssaal zu sehen ist. Hierzu folgten Artikel über die hiesige Meteoritensammlung, die Ausarbeitung eines Kataloges über die in den Sammlungen der DDR vorhandenen Meteorite, die wissenschaftliche Erstbearbeitung eines neuen nickelreichen Eisenmeteoriten aus Thüringen und die intensive wissenschaftsgeschichtliche Aufarbeitung der Leistungen und Ideen des Physikers E. F. F. Chladni als Begründer der Meteoritenkunde. Die von Günter Hoppe am Museum für Naturkunde durchgeführte wissenschaftliche Tagung aus Anlass des 150. Todestages Chladnis führte wissenschaftshistorische Betrachtungen und moderne Erkenntnisse der Meteoritenforschung zusammen. Analog verlief auch eine Tagung zu Ehren von C. S. Weiss am Museum sehr erfolgreich. Günter Hoppe initiierte zahlreiche weitere Ausstellungen, so z. B. zeitweilige Ausstellungen über neu erworbene Sammlungen, zur Mitwirkung von Sammlergruppen oder zu besonderen historischen Anlässen und Personen. Darunter eine hervorragende Ausstellung über die Reise Alexander von Humboldts und seines Begleiters Gustav Rose in den Ural und den Altai 1829. Hohe Anerkennung und Beachtung als ständige Ausstellung im Mineralsaal erreicht das Ensemble dreier Vitrinen, die dem Wirken des Chemikers Martin Heinrich Klaproth, dem Kristallographen Christian Samuel Weiss und dem Mineralogen Gustav Rose gewidmet sind. Hier wird dem Museumsbesucher in anregender Weise ein Teil Wissenschaftsgeschichte nahe gebracht.

Noch umfangreicher und vielfältiger zeigen sich die Ergebnisse der von Günter Hoppe als Hauptaufgabe empfundenen und mit großer Sorgfalt und Feingefühl betriebenen wissenschaftshistorischen Studien zu diesen und weiteren bedeutenden Persönlichkeiten, wie Leopold von Buch und Dietrich Ludwig Gustav Karsten, in den zahlreichen wissenschaftlichen
Publikationen. Diese Arbeiten wurden nach dem Ende der Amtszeit im Herbst 1984, darin auch in den Jahren 1977 bis 1981 als Direktor des Museums für Naturkunde tätig, in anerkennenswerter Weise fortgesetzt. Ein anderes von Günter Hoppe angeregtes Projekt über die Minerale der DDR, das historische und aktuelle Kenntnisse verbinden sollte, bereitete ihm zwar sehr viel Arbeit, konnte aber leider nicht zum Erfolg geführt werden.

Auch noch lange nach seiner Emeritierung im Jahre 1984 war er dazu zu bewegen, sich der Geschichte der Geowissenschaften am Museum und ganz allgemein in Berlin zu widmen. Mit der für ihn so typischen Zurückhaltung erklärte er sich bereit, in der 1998 neu gegründeten Geowissenschaftlichen Reihe der Mitteilungen des Museums für Naturkunde eine Serie über die Geowissenschaften in Berlin von seinen Anfängen im 16. Jahrhundert bis 1910 zu schreiben. Jedes Jahr pflegte er auf sein Alter hinzuweisen und seine Zweifel vorzubringen, die Serie zum Abschluss bringen zu können. Es ist ihm dennoch gelungen, fünf Teile einer Serie dort abzuschließen, wo viele der Berichte über die Geowissenschaften des Museums enden - 1910, dem hundertjährigen Jubiläum der Humboldt-Universität (siehe Beitrag von Schultze zur Geschichte der Geowissenschaften in diesem Band). Alle Beiträge Hoppe's zeugen von dem Bestreben, die Ereignisse so wahrheitsgetreu wie möglich aufgrund erschließbarer Dokumente darzulegen. Man muss schon genau hinsehen, um den Hinweis auf eine Fehldarstellung eines anderen Autors zu finden. Herr Hoppe scheut sich davor, seine persönlichen Auffassungen oder Kritik auch nur anklingen zu lassen, daher wird er sich auch nicht der jüngeren Geschichte stellen, da damit zu viele Emotionen verbunden sind.

Günter Hoppe war und ist beliebt und geschätzt wegen seiner menschlichen Wärme, die ihm auch in führenden Positionen unter politisch ungünstigen Umständen nicht abhanden kam. Sich selbst betreffend zeigt er eine Bescheidenheit, die fast bis zur Selbstverleugnung geht. Von unauffälliger Großzügigkeit gegenüber anderen scheut er vor jeder Herausstellung seiner Person zurück. Es ist dies ein seltener Charakterzug von Personen einer so großartigen Lebensleistung.

\section{Publikationen}

(zusammengestellt von G. Hoppe)

Das hier vorgestellte Schriftenverzeichnis zeigt die zwei Phasen der Aktivitäten von G. Hoppe: die wissenschaftliche Beschäftigung mit mineralogischen Objekten von 1951 bis 1971, 
gefolgt von der Befassung mit der Geschichte der Mineralogie und seiner Repräsentanten von 1970 bis 2004 .

1951. Die akzessorischen Schwermineralien in Graniten und verwandten Gesteinen des Harzes. - Fortschritte der Mineralogie 29/30: 72-77.

1951. Die akzessorischen Schwermineralien in Eruptivgesteinen am Beispiel des Ramberggranites und anderer Harzer Gesteine. - Geologica 9: 1-114. (Dissertation Halle 1950).

1957. Das Erscheinungsbild der akzessorischen Zirkone des Lausitzer Granodiorits von Wiesa bei Kamenz und seine petrogenetische Auswertung. - Geologie 6: 289-305.

1957. [Über Tonforschung in Deutschland in den letzten Jahren. - Materialy soweschtschanija po issledowaniju i ispolsowaniju glin, Lwow: 802-810. [Übersetzung in das Russische von E. I. Wultschyn].

1958. Eine Tontagung in Lwow (Sowjetunion). - Geologie 7: 209-217.

1958. Präparationsmethode zur Untersuchung von Mineralkörnern mit Kernemulsion. - Naturwissenschaften 45 : 206.

1959. Über die Verwendbarkeit der akzessorischen Zirkone zu Altersbestimmungen. - Neues Jahrbuch für Mineralogie, Abhandlungen 93: 45-66.

1959. Zur Frage des Auftretens übergroßer radioaktiver Höfe im Arnögranit. - Geologiska Föreningens Förhandlingar, Stockholm 81: 485-494.

1959. Die Schwermineralien der tertiären Sande des Geiseltales. - Geologie 8: 612-624.

1960. Ein pneumatisches Auslesegerät für feine Partikel. Zeitschrift für angewandte Geologie 6: 515-516.

1961. Zur Morphologie des akzessorischen Zirkons. - Berichte der Geologischen Gesellschaft in der DDR 6: $27-41$.

1962. Petrogenetisch auswertbare morphologische Erscheinungen an akzessorischen Zirkonen. - Neues Jahrbuch für Mineralogie, Abhandlungen 98: 35-50.

1962. Die akzessorischen Zirkone aus dem Granitkomplex der Lausitz (Sachsen). - Freiberger Forschungshefte, Reihe C 129: 35-50.

1962. Die Formen des akzessorischen Apatits. - Berichte der Geologischen Gesellschaft in der DDR 7: 233-239.

1962. Die akzessorischen Zirkone aus Gesteinen des Bergeller und des Adamello-Massivs. - Chemie der Erde 22. $245-263$.

1962. [Zum Auftreten radioaktiver Höfe im Biotit.] - Mineralogitscheskij Sbornik Lwowskogo geologitscheskogo Obschestwa 16: 328-333. [Russisch]

1963. Die Verwendbarkeit morphologischer Erscheinungen an akzessorischen Zirkonen für petrogenetische Auswertungen. - Abhandlungen der Deutschen Akademie der Wissenschaften zu Berlin, Klasse für Bergbau, Hüttenwesen und Montangeologie, (1963) Nr.1: 130 pp. - (Habilitationsschrift Halle 1960).

1963. Identifizierung von Tuff mit Hilfe des Zirkons und Nachweis von Quarzporphyrtuff des Auerberges (Harz). - Naturwissenschaften 50: 688.

1964. Zirkone aus Gesteinen des mitteldeutschen Permokarbons. I. Zirkone aus Sedimenten und Tuffen der Umgebung von Wettin (Hallescher Permokarbonkomplex). - Geologie 13: 1059-1079. (mit Schwab, M.)

1964. Morphologische Untersuchungen als Beiträge zu einigen Zirkon-Altersbestimmungen. - Neues Jahrbuch für Mineralogie, Abhandlungen 102: 89-106.

1965. Zirkone aus Gesteinen des mitteldeutschen Permokarbons. II. Die Altersstellung des Auerbergporphyrs im Harz. - Geologie 14: 777-813. (mit Kunert, R. \& Schwab, M.)

1965. Morphologische Untersuchungen als Beiträge zu Zirkon-Altersbestimmungen. 2. Mitteilung. - Neues Jahrbuch für Mineralogie, Abhandlungen 103: 273-285.
1966. Zirkone aus Granuliten. - Berichte der Deutschen Gesellschaft für Geologischen Wissenschaften, Reihe $B$, Mineralogie und Lagerstättenforschung 11: 47-81.

1966. Zur Lichtbrechung von Montmorillonit mit organischen Zwischenschichten. - Naturwissenschaften 53: 104-105. (mit Landgraf, K.-F.)

1966. Nachweis der pyroklastischen Entstehung der Kohlentonsteine von Oelsnitz / Sachsen. - Berichte der Deutschen Gesellschaft für Geologischen Wissenschaften, Reihe B, Mineralogie und Lagerstättenforschung 11: 215-222.

1966. [Probleme der Morphologie akzessorischer Zirkone.]Mineralogitscheskij Sbornik Lwowskogo Universiteta. 26: 554-558. [Russisch]

1967. Die Zirkone eines pyroklastischen Gesteins aus dem Silur von Hasselfelde (Harz). - Geologie 16: 542-547.

1967. Zirkone aus pyroklastischen und klastischen Gesteinen des Kohlenbeckens von Kladno-Slany-Rakovník (mittelböhmisches Oberkarbon). - Berichte der Deutschen Gesellschaft für Geologischen Wissenschaften, Reihe $B$, Mineralogie und Lagerstättenforschung 12: 145-164. (mit Masek, J.)

1968. Probleme der Paragenese von Tonmineralen am Beispiel der Tonsteine. - Freiberger Forschungshefte, Reihe C, 231: 169-173.

1968. (Zu Fragen der Beziehungen von Eruptivgesteinen und organischen Bestandteilen von Gesteinen im Hinblick auf die Kohlenwasserstoff-Höffigkeit, bezogen auf Verhältnisse im Norden der DDR. - Unveröffentlichter Bericht).

1969. Ein Verfahren zum Nachweis der Verlagerungsfähigkeit von Tonmineralen. - Berichte der Deutschen Gesellschaft für Geologischen Wissenschaften, Reihe B, Mineralogie und Lagerstättenforschung 14: 43-47. (mit Helmchen, H.)

1969. Zur Verlagerung von Tonmineralen durch strömende Lösungen. - Berichte der Deutschen Gesellschaft für Geologischen Wissenschaften, Reihe B, Mineralogie und Lagerstättenforschung 14: 203--218. (mit Helmchen, H.)

1969. Die Entwicklung der mineralogischen Wissenschaften in den 20 Jahren seit Bestehen der DDR. - Geologie 18: 959-982. (mit Kaemmel, Th. \& Engel, A.)*

1969. Katalog der Meteoriten aus dem Museum für Naturkunde an der Humboldt-Universität Berlin, Mineralogisches Museum. - Berichte der Deutschen Gesellschaft für Geologischen Wissenschaften, Reihe B, Mineralogie und Lagerstättenforschung 14: 359-381. (mit Wappler, G.)

1970. Zum Problem der pigmentierten akzessorischen Apatite. - Berichte der Deutschen Gesellschaft für Geologische Wissenschaften, Reihe B, Mineralogie und Lagerstättenforschung 15: 33-54.

1970. Die Meteoritensammlung des Mineralogischen Museums der Humboldt-Universität Berlin. - Wissenschaftliche Zeitschrift der Humboldt-Universität zu Berlin, Mathematisch-naturwissenschaftliche Reihe 19: 128-138.*

1971. Zur Erfassung der Meteorite. - Chemie der Erde 30: 191-197.

1971. Meteorite als Informationsträger. - Astronomie und Raumfahrt, Jahrgang 1971: 136-139.

1971. Meteoritenbergung durch die Bevölkerung - kosmische Materie kostenlos. - Wissenschaft und Fortschritt 21: $527-528$.

1971. Geschichte des Mineralogischen Museums im Museum für Naturkunde an der Humboldt-Universität Berlin. - Fundgrube 8: 74, 79-82.*

1972. Neuerwerbungen des Mineralogischen Museums Berlin (Sammlungen Max Köhler und Curt Gerber). - Fundgrube 9: 3. Umschlagseite von Heft 1/2.*

1972. Geschichte des Mineralogischen Museums im Museum für Naturkunde an der Humboldt-Universität Berlin. - Fundgrube 9: 20, 29-30* 
1974. Das Baumaterial. - In Hohl, R. (Hrsg.). Unsere Erde. Eine moderne Geologie: 45-64, Urania-Verlag. Leipzig. (2. Aufl. 1977; 3. Aufl. 1984).

1974. Der Meteoritenfall am 4. 10. 1973 im Bezirk Erfurt. Astronomie und Raumfahrt, Jahrgang 1974: 143-146.

1975. Gesamtkatalog der in der Deutschen Demokratischen Republik vorhandenen Meteorite. - Wissenschaftliche Zeitschrift der Humboldt-Universität zu Berlin, Mathematisch-naturwissenschaftliche Reihe 24: 521-569.*

1976. Der Eisenmeteorit von Dermbach, ein neuer Ni-reicher Ataxit. - Chemie der Erde 35: 305-316.

1976. Mineralogische Forschungsergebnisse Gustav Roses von der Rußlandreise mit Alexander von Humboldt (1829). - Zeitschrift für geologische Wissenschaften 4: 337-344. (mit Wappler. G.)*

1976. Das Pallas-Eisen, ein Ausgangspunkt für die Meteoritentheorie E. F. F. Chladnis (1794). - Zeitschrift für geologische Wissenschaften 4: 521-528.*

1976. Ein Dokument über die Tätigkeit des armenischen Mineralogen A. Arzruni in Berlin. - Ajreniki Dsajn (.,Stimme der Heimat". Zeitung in armenischer Sprache) vom 21. 1. 1976.*

1976. Eine mineralogische Exkursion zum Ural und Altai vor 150 Jahren. - Fundgrube 12: 52-58. (mit Wappler, G.)*

1976. Sonderausstellungen der letzten Jahre im Museum für Naturkunde Berlin. - Fundgrube 12: 94--96. (mit Barthel, M.)*

1977. Meteorite, Materie des Kosmos. - Faltblatt des Museums für Naturkunde Berlin, 12 pp.

1977. Das Pallas-Eisen. - Neue Museumskunde 20: 2-3.*

1977. Ernst Florens Friedrich Chladni. Zum 150. Todestag des Begründers der Meteoritenkunde. - Chemie der Erde 36: $249-262$. $^{*}$

1977. Musik und Meteoriten. E. F. F. Chladni - ein Naturforscher aus Wittenberg. - Wochenpost 24. Nr. 28 vom 8. 7. 1977: $16 . *$

1978. Goethes Ansichten über Meteorite und sein Verhältnis zu dem Physiker E. F. F. Chladni. - Goethe-Jahrbuch 95: $227-240$.*

1979. E. F. F. Chladni. Über den kosmischen Ursprung der Meteorite und Feuerkugeln (1794). Mit Erläuterungen von G. Hoppe. - In: Ostwalds Klassiker der exakten Wissenschaften, Heft 258: 103 pp. Geest \& Portig. Leipzig (2.verbesserte Aufl. 1982, 104 pp.)*

1980. Die stoffliche Entwicklung kosmischer Festkörper. In Vent, W. (Hrsg.). Beiträge zu Prinzipien und Problemen der Systematik und Evolutionsforschung aus dem Museum für Naturkunde: 9-18. Humboldt-Universität. Berlin.

1980. Der „Meteorit" von Ichstedt ist kein Meteorit. - Astronomie und Raumfahrt 18: 13

1980. Glaskopfförmiger Goethit aus Wüstensand von Wau en-namus, Libyen. - Fundgrube 16: 45-47, 51.

1980. Zum 200. Geburtstag des Berliner Mineralogen und Kristallographen Christian Samuel Weiss. - Fundgrube 16: $126-127$ * $^{*}$

1982. Meteorite - Erscheinung. Beschaffenheit und Entwicklung. - Die Sterne 58: 352-364.

1982. Friedrich Fröbel als Mineraloge. - Fundgrube 18: $8-10$.*

1982. Christian Samuel Weiss und das Berliner Mineralogische Museum. - Wissenschaftliche Zeitschrift der Humboldt-Universität zu Berlin, Mathematisch-naturwissenschaftliche Reihe 31: $245-254$.*

1982. Regeln für Besucher von Museen vor 250 Jahren. Fundgrube 18: $30-31$.*

1982. (Die Zusammenarbeit J. C. L. Zinkens (1790-1862) mit Mineralogen und Chemikern der Universität Berlin. - Unpublizierter Vortrag).*

1983. Ein Museumsdiebstahl vor 144 Jahren. - Neue Museumskunde 26: 20 *

1983. Die Mineralsammlung Martin Heinrich Klaproths und seine mineralanalytischen Bestrebungen. - Zeitschrift für geologische Wissenschaften 4: 1245-1253. (mit Wappler, G.)*

1984. Der Anteil Dietrich Ludwig Gustav Karstens an der Entwicklung der Mineralsystematik. - Zeitschrift für geologische Wissenschaften 12: 709--717.*

1984. Alexander von Humboldts Einstellung zum Sammeln. - Fundgrube 20: 98-101. *

1985. Dietrich Ludwig Gustav Karsten (1768-1810). Mineraloge und Bergbeamter in Preußen. - In Prescher, $\mathrm{H}$. (Hrsg.). Leben und Wirken deutscher Geologen im 18. und 19. Jahrhundert: 71-92. Verlag für Grundstoffindustrie, Leipzig.*

1986 (für 1984). Die Beziehungen von Baron Sigmund Zois (1747-1819) zu Berliner Naturforschern. - Geologija, Ljubljana 27: 27-38.*

1986. Der Beitrag Alexander von Humboldts zur Entwicklung der geowissenschaftlichen Sammlungen der Berliner Universität. - Abhandlungen der Akademie der Wissenschaften der DDR, Abteilung Mathematik, Naturwissenschaften, Technik, Jahrgang 1985, Nr. 2 N: 92-97. (Auch in: A. v. Humboldt-Ehrung in der DDR: 99-105. Akademie-Verlag, Berlin). (mit Barthel, G.)*

1986. Das historische Buttermilcherz von Adreasberg/Harz (BRD), ein Gemenge von Chlorargyrit und Schichtsilikaten. - Chemie der Erde 45: 147-158. (mit Damaschun, F.)*

1987. Eine Würdigung Martin Heinrich Klaproths als Mineralchemiker. - Die Pharmazie, Berlin 42: 266-267. (mit Damaschun, F. \& Wappler, G.)*

1987. Zum Meteoritenfall von Niederreißen (1581). - In Grausame und schreckliche Neuigkeiten. Herausgeber: Nationale Forschungs- und Gedenkstätten der Klassischen Deutschen Literatur, Weimar: 6-8.*

1987. Zum Anteil Berlins an der Entwicklung der mineralogisch-geologischen Wissenschaften bis zur Mitte des 19. Jahrhunderts (Vortrag 1984). - WissenschaftlichTechnischer Informations-Dienst, Zentrales Geologisches Institut, Berlin 28, A 1: 15-19.*

1987. Die Meteoritensammlung E. F. F. Chladnis. - Die Sterne 63: $315-329 . *$

1987. Das Königliche Mineralienkabinett in Berlin. Vorläufer des Mineralogischen Museums der Berliner Universität. - Neue Museumskunde 30: 295-307.*

1987. Eisen von Disko. - Fundgrube 23: S. 25-26.*

1987. Zur Mineralfälschung, eine Ergänzung. - Aufschluß 38. Beilage VFMG aktuell: 27.*

1988. Das Buttermilcherz. - Fundgrube 24: 38-42. (Auch nicht gestatteter Abdruck in: Emser Hefte 10, 2: 33-37). (mit Otto, H.)*

1989. D. L. G. Karstens Museum Leskeanum, die früheste publizierte Anwendung der Lehre A. G. Werners auf eine Sammlung (Vortrag 1986). - Abhandlungen und Berichte aus dem Naturkundemuseum Görlitz 60, 2: 72-78.*

1989. Martin Heinrich Klaproth (1743-1817) als Mineralchemiker und Mineralsammler. - Aufschlu 3 40: 201-214.*

1989. Ernst Florens Friedrich Chladni (1756-1827). Akustiker und Begründer der Meteoritenkunde. - Katalog der Mineralientage München 1989: 48-53.*

1989. Alexander von Humboldt und die Berliner Mineralogie. - Wissenschaftliche Zeitschrift der Humboldt-Universität zu Berlin, Mathematisch-naturwissenschaftliche Reihe 38: 308-318.*

1989. Aus der Geschichte des Berliner Mineralogischen $\mathrm{Mu}-$ seums. Gedanken zu einem Ausstellungsensemble. Wissenschaftliche Zeitschrift der Humboldt-Universität zu Berlin, Mathematisch-naturwissenschaftliche Reihe 38: $319-325 *$

1989. Die mineralogische Ausstellung im Museum für Naturkunde der Humboldt-Universität. - Wissenschaftliche Zeitschrift der Humboldt-Universität zu Berlin, Mathematisch-naturwissenschaftliche Reihe 38: $326-330$. (mit Wappler, G. \& Wäsch, E.)* 
198'. Pechblende und andere Uranminerale von Johanngeorgenstadt - Originalobjekte der Uranentdeckung. Neue Museumskunde 32: 242-243.*

1989. M. H. Klaproth, C. S. Weiss und G. Rose, ein biographisches Ensemble in der mineralogischen Ausstellung des Museums für Naturkunde in Berlin. - Neue Museumskunde 32: 261-267. (mit Wappler, G. \& Damaschun, F.) ${ }^{*}$

1987. Martin Heinrich Klaproth (1743-1817), ein großer Sohn Wernigerodes. - Der Harz, Wernigerode 21: $81-85$.* $^{*}$

1990. Johann Jakob Ferber (1743-1790) und die Gesellschaft naturforschender Freunde in Berlin. - Fundgrube 26: $2-7 . *$

1990: Alexander von Humboldt und die Entdeckung des Vanadiums. - Chemie der Erde 50: 81-94. (mit Siemroth, J. \& Damaschun, F.)*

1960. Gedenkstein für Gustav Rose (1798-1873). - Fundgrube 26: $160-161$.* $^{*}$

1950. Karl Ludwig Giesecke. Ein preußischer Bergrat (?) auf Grönland 1806-1813. - Fundgrube 26: 109-114.*

19!1. Karl Ludwig Giesecke $(1761-1833)$ und Berlin. Aufschluß 42: 53-63.*

1991. Ordnung ins Mineralreich. (Eine Betrachtung aus Anlaß des 50 jährigen Jubiläums der „Mineralogischen Tabellen" von Hugo Strunz). - Katalog der Mineralientage München 1991: 98-102. (mit Wappler, G.)*

19'2. Einige Gedenktage des Jahres 1992. - Fundgrube 28: $2-5$. $^{*}$

19 2. Abraham Gottlob Werner (1749-1817) und Berlin. Aufschluß 44: 257-266.*

1972. Der Donnerstein von Ensisheim. Zum 500-jährigen Jubiläum des berühmten Meteoritenfalls. - MuseumsJournal, Berlin 6, 4: 55-57.*

1993. Berlin im Jahre 1793. Das Tagebuch Adolf Traugott v. Gersdorfs. - Der Bär von Berlin. Jahrbuch des Vereins für die Geschichte Berlins 42: 7-47.*

1993. Martin Heinrich Klaproth (1743-1817). Zum 250. Geburtstag des bedeutenden Berliner Pharmazeuten und Chemikers. - Mitteilungen des Vereins für die Geschichte Berlins 89: 189-197.*

1994. Die Entwicklung der Ansichten Alexander von Humboldts über den Vulkanismus und die Meteorite. - In: Studia Fribergensia. A.-v.-Humboldt-Kolloquium Freiberg 1991. Beiträge zur A.-v.-Humboldt-Forschung, Berlin 18: $93-113 . *$

1994. Das Schicksal von Martin Heinrich Klaproths Ruhestätte. - In Engel, M. (Hrsg.): Von der Phlogistik zur modernen Chemie. Symposium zum 250. Geburtstag von Martin Heinrich Klaproth, TU Berlin 1993, Berlin: 21-29.*

1995. Henrik Christian Ström (1784-1836). Schöpfer eines Bildnisses von Abraham Gottlob Werner. - Nachrichtenblatt zur Geschichte der Geowissenschaften 3 (für 1993): $30-32 . *$

1995. Johann Jacob Ferber (1743-1790). Zum Leben und Wirken des bedeutenden Geo- und Montanwissenschaftlers. - Aufschluß 46: 233--244.*

1996. Georgius Agticola (1494-1555) und Abraham Gottlob Werner (1749-1817). Eine vergleichende Biographie aus dem Jahre 1819 von Friedrich Liebegott Becher. Erstmalig vollständig, mit dem von der Zensur verbotenen Nachtrag herausgegeben, kommentiert und mit Anmerkungen versehen. X, $127 \mathrm{pp}$. Verlag für Wissenschafts- und Regionalgeschichte Dr. Michael Engel, Berlin. (Hrsg. mit Otto, H.)*

1998. Die Bildnisse Leopold von Buchs. - Geohistorische Blätter. Zeitschrift des Vereins Berlin-Brandenburgische Geologie-Historiker „Leopold von Buch" 1: 7-14.*

1998. Das Institut für Mineralogie und seine Sammlungen im Museum für Naturkunde der Humboldt-Universität zu Berlin. - Aufschluß 49: 261-276. (Wappler, G.)*

1998. Zur Geschichte der Geowissenschaften im Museum für Naturkunde zu Berlin. Teil 1. Aus der Vorgeschichte bis zur Gründung der Berliner Bergakademie im Jahre 1770. - Mitteilungen aus dem Museum für Naturkunde der Humboldt-Universität in Berlin, Geowissenschaftliche Reihe 1: 5-19.*

1998. Pabst von Ohains Sammlung kam nicht nach Berlin. -Fundgrube 34: 56-60.*

1998. Die Briefe im Nachlaß Leopold von Buchs (1774-1853). Mit drei Briefen an L. v. Buch von J. F. L. Hausmann, S. H. Spiker und K. F. P. v. Martius. - Geohistorische Blätter, Zeitschrift des Vereins Berlin-Brandenburgische Geologie-Historiker „Leopold von Buch" 1: 115-129. (mit Landsberg, H.)*

1999. Die Konstruktion des Erdballs" - Humboldt und die Geologie. - In Katalog zur Ausstellung Alexander von Humboldt. Netzwerke des Wissens. Hrsg. Kunstund Ausstellungszentrum der Bundesrepublik Deutschland $\mathrm{GmbH}: 93 . *$

1999. Zwei Briefe Leopold von Buchs an D. L. G. Karsten. Zum 225. Geburtstag L. v. Buchs. - Geohistorische Blätter, Zeitschrift des Vereins Berlin-Brandenburgische Geologie-Historiker "Leopold von Buch" 2: $33-40 . *$

1999. Leopold von Buchs Verhältnis zu Abraham Gottlob Werner (nach unveröffentlichten Tagebüchern und Briefen L. v. Buchs). Vortrag D 02, Kurzfassung. - Tagungsband der Tagung „Abraham Gottlob Werner und seine Zeit", Freiberg.*

1999. Zur Geschichte der Geowissenschaften im Museum für Naturkunde zu Berlin. Teil 2. Von der Gründung der Bergakademie zur Gründung der Universität 1770-1810. - Mitteilungen aus dem Museum für Naturkunde der Humboldt-Universität in Berlin, Geowissenschaftliche Reihe 2: 3-24.*

1999. Die Tagebücher Leopold von Buchs. Chronologische Übersicht. - Geohistorische Blätter. Zeitschrift des Vereins Berlin-Brandenburgische Geologie-Historiker "Leopold von Buch“" 2: 107-115.*

2000. Friedrich Hessenberg (1810-1874) - Juwelier, Mineraloge und Mineralsammler. - Aufschluß 51: 259-293.*

2000. Zur Geschichte der Geowissenschaften im Museum für Naturkunde zu Berlin. Teil 3: Von A. G. Werner und R. J. Haüy zu C. S. Weiss - Der Weg von C. S. Weiss zum Direktor des Mineralogischen Museums der Berliner Universität. - Mitteilungen aus dem Museum für Naturkunde der Humboldt-Universität in Berlin, Geowissenschaftliche Reihe 3: 3-25.*

2000. Eine Berliner Preisfrage zum Basaltproblem vom Anfang des 19. Jahrhunderts - Das letzte Wort von J. C. W. Voigt zum Neptunismus-Vulkanismus-Streit. Geohistorische Blätter. Zeitschrift des Vereins BerlinBrandenburgische Geologie-Historiker "Leopold von Buch" 3: 101-114.*

2001. Eine Berliner Preisfrage zum Basaltproblem vom Anfang des 19. Jahrhunderts - Das letzte Wort von J. C. W. Voigt zum Neptunismus-Vulkanismus-Streit. Geohistorische Blätter. Zeitschrift des Vereins BerlinBrandenburgische Geologie-Historiker ,Leopold von Buch" 4: 1-20.*

2001. Leopold von Buch (1774-1853). Der bedeutendste deutsche Geologe seiner Zeit. - Katalog der Ausstellung „Marksteine. Eine Entdeckungsreise durch Brandenburg-Preußen." des Hauses der BrandenburgischPreußischen Geschichte Potsdam 18.8.-11.11.2001, Berlin: $378-380$.* $^{*}$

2001. Zur Geschichte der Geowissenschaften im Museum für Naturkunde zu Berlin. Teil 4: Das Mineralogische $\mathrm{Mu}$ seum der Universität Berlin unter Christian Samuel Weiss von 1810 bis 1856 . - Mitteilungen aus dem $\mathrm{Mu}$ seum für Naturkunde der Humboldt-Universität in Berlin, Geowissenschaftliche Reihe 4: 3-27.*

2001. Die Beziehungen zwischen E. E. F. v. Schlotheim und D. L. G. Karsten (nach den Briefen von Karsten an Schlotheim). - Mitteilungen aus dem Museum für Na- 
turkunde der Humboldt-Universität in Berlin. Geowissenschaftliche Reihe 4: 29-35.*

2002. Leopold von Buchs Verhältnis zu Abraham Gottlob Werner. Mit Regesten der Briefe v. Buchs an Werner und Auszügen aus dem Tagebuch v. Buchs des Jahres 1804. - Symposium ,Abraham Gottlob Werner und die Begründung der Geowissenschaften" in Freiberg, September 1999. - Freiberger Forschungshefte, Reihe D, Montan- und Technikgeschichte 207: 124-135.* [Das Literaturverzeichnis wurde nicht gedruckt.]

2002. Peter S. Pallas und das Pallas-Eisen - Aus der Geschichte der Metcoritenkunde. - Theatrum naturae. Hrsg. Fördererkreis der naturwissenschaftlichen Museen Berlins e. V. 2002, Heft 2:16-17. - [Redaktionell sehr stark gekürzt und verändert.].*

2002. Peter Simon Pallas und das Pallas-Eisen. - Ein frühes Kapitel aus der Geschichte der Meteoritenkunde. Geohistorische Blätter. Zeitschrift des Vereins BerlinBrandenburgische Geologie-Historiker ..Leopold von Buch" 5: 99-108.*

2002. Student bei Ferdinand von Wolff. Zum Gedenken an meinen Lehrer anlässlich seines Todestages vor $50 \mathrm{Jah}$ ren. - Geohistorische Blätter. Zeitschrift des Vereins Berlin-Brandenburgische Geologie-Historiker ..Leopold von Buch" 5: 151-161.*

2003. Friedrich Anton von Heinitz (Heynitz) (1725-1802). - Nachrichtenblatt zur Geschichte der Geowissenschaften 13: $61-73 . *$
2003. Gustav Rose, Alexander von Humboldt und die Berliner Mineralogie in der ersten Hälfte des 19. Jahrhunderts. - In Hamel, J., Knobloch, E. \& Pieper, H. (Hrsg.). Alexander von Humboldt in Berlin. Sein Einfluss auf die Entwicklung der Wissenschaften. Beiträge zu einem Symposium. - Algorismus. Studien zur Geschichte der Mathematik und der Naturwissenschaften. Hrsg. Menso Folkerts, München 41: 223-236. (mit Suckow, Ch.)*

2003. Zur Geschichte der Geowissenschaften im Museum für Naturkunde zu Berlin. Teil 5: Vom Mineralogischen Museum im Hauptgebäude der Universität Berlin zu den zwei geowissenschaftlichen Institutionen im $\mathrm{Mu}-$ seum für Naturkunde - 1856 bis 1910 . - Mitteilungen aus dem Museum für Naturkunde der Humboldt-Universität in Berlin, Geowissenschaftliche Reihe 6: $3-51$.*

2004. Friedrich Tamnau (1802-1879), Mineraloge, Mineralsammler und Mäzen. - Mitteilungen aus dem Museum für Naturkunde der Humboldt-Universität in Berlin, Geowissenschaftliche Reihe 7: 45-60.*

(Im Druck). Gustav Rose, der Reisebegleiter von A. v. Humboldt 1829. - In Aranda, K., Förster, A. \& Suckow, Ch. (Hrsg.). DAMU-Expeditions- und Konferenzbuch .Auf den Spuren Alexander von Humboldts in Russland"**

* Teilweise oder ganz wissenschaftshistorisch. 